\title{
X. On Sir D. Brewster's deductions from the hourly observations at Leith in 1824-25
}

\section{S.M. Drach Esq. F.R.A.S.}

To cite this article: S.M. Drach Esq. F.R.A.S. (1842) X. On Sir D. Brewster's deductions from the hourly observations at Leith in 1824-25, Philosophical Magazine Series 3, 21:135, 43-46, DOI: 10.1080/14786444208621474

To link to this article: http://dx.doi.org/10.1080/14786444208621474

册 Published online: 01 Jun 2009.

Submit your article to this journal $[\pi$

Џll Article views: 2

Q View related articles 두 


\section{Mr. Drach on the Hourly Observations at Leith in 1824-25. 43}

This process is cheaper than that of heating chlorate of potash ; for two parts of bichromate of potash will produce as much oxygen as one of chlorate of potash, while the latter is nearly three times the price of the former; and besides this, the residue of the first is valuable, and may be reconverted into bichromate of potash. It is likewise a more convenient process than any at present known, since it may be conducted at so low a temperature that an ordinary retort and lamp may be used for the production of a considerable quantity of oxygen.

Mechanics' Institution, Liverpool, May 10, 1842.

W. H. Balmain.

[Note. - I have tried this process and find that it answers very well, the gas being given off, I think, with greater readiness than when sulphuric acid and binoxide of manganese are employed. Occasions I have no doubt will occur in which this method may be advantageously substituted for others.R. P.]

X. On Sir D. Brewster's Deductions from the Hourly Observations at Leith in 1824-25. By S. M. DRACH, Esq., F.R.A.S.

To the Editors of the Philosophical Magazine and Journal. Gentuemen,

THE deductions alluded to in the title of this article, as detailed in the Edinburgh Philosophical Transactions, vol. $x$., flow from any expression of the temperature in functions of the time. Let $v=$ the temperature, $t=$ the time; $-T=$ a fixed instant; then to be real $v=$ function of

$$
\left\{\overline{t+T}{ }^{i}, \quad(t+\mathrm{T})^{i}, \log (t+\mathrm{T}),{ }_{\cos }^{\sin } i(t+\mathrm{T}), \text { constant }\right\},
$$

which is developable into the series

$$
v=\mathrm{A}+\mathrm{B}(t+\mathrm{T})+\mathbf{C}(t+\mathrm{T})^{2}+\mathbf{D}(t+\mathrm{T})^{3}+\& \mathrm{c} .
$$

$A, B, C, \& c$ are functions independent of the time, and comprehending the latitude, declination, radiation, \&c.

When $t=-\mathrm{T}, v=\mathrm{A}$.

First. If $\mathrm{A}=$ the daily mean temperature, $t=-\mathrm{T}=$ time of morning mean, and $0=\mathrm{B}+\mathrm{C}(t+\mathrm{T})+\mathrm{D}(t+\mathrm{T})^{2},+\& \mathrm{c}$. gives the other times of mean daily temperature.

There being only one (evening) mean, this series must, be very convergent, and

$$
t=-\mathrm{T}-\frac{\mathbf{B}}{\mathbf{C}}, \text { or more correctly, } t=-\mathbf{T}-\frac{\mathbf{B}}{\mathrm{C}-\frac{\mathrm{B}}{\mathrm{C}} \cdot \mathbf{D}} ;
$$

thus $B$ D is very much less than $C^{2}$. 
Secondly. For the maximum and minimum times:

$$
\begin{aligned}
& \frac{d v}{d t}=0=\mathrm{B}+2 \mathrm{C}(t+\mathrm{T})+3 \mathrm{D}(t+\mathrm{T})^{2}, \\
& \because t=-\mathrm{T}-\frac{\mathrm{C}}{3 \mathrm{D}}+\sqrt{\frac{\mathrm{C}^{2}-3 \mathrm{BD}}{9 \mathrm{D}^{2}}}
\end{aligned}
$$

the first corresponds to a minimum, the second to a maximum ; the former being nearer than the latter to the morning mean.

Thirdly. If $\mathrm{A}, \mathrm{T}$ be the temperature and epoch, and $t$ not great,

$v=\left(\begin{array}{c}\mathbf{A}+\mathbf{B} \mathbf{T}+\mathbf{C T}^{2} \\ +\mathbf{D ~ T}^{3}\end{array}\right)+\left(\begin{array}{c}\mathbf{B}+2 \mathbf{C T} \\ +3 \mathbf{D T}^{2}\end{array}\right) t+\left(\mathbf{C}^{2}+3 \mathbf{D T}\right) t^{2}+\mathbf{D} \cdot t^{3}$

is the equation for some time on each side of $T$; neglecting the small quantity $\mathrm{D} t^{3}$, it is that of a parabola, having $v$ for an absciss and $t$ for an ordinate.

Fourthly. Beginning at noon, $\mathrm{T}=0, v=\mathbf{A}+\mathbf{B} t+\mathbf{C} t^{2}$ $+\mathrm{D} t^{3}+\& \mathrm{c}$. Taking the mean of homonymous hours (the unit of $t$ being one day), that is, taking the mean of $t+\frac{1}{4}$ and $t-\frac{1}{4}$, we obtain

$$
\begin{array}{r}
v_{t+4}=\mathrm{A}+\mathrm{B}\left(t+\frac{1}{4}\right)+\mathrm{C}\left(t^{2}+\frac{t}{2}+\frac{1}{16}\right)+8 \mathrm{c} \\
=\mathrm{A}+\frac{\mathrm{B}}{4}+\frac{\mathrm{C}}{16}+\left(\mathrm{B}+\frac{\mathrm{C}}{2}\right) t+\mathrm{C} t^{2}+8 \mathrm{c} \\
v_{t-\frac{1}{4}}=\mathrm{A}-\frac{\mathrm{B}}{4}+\frac{\mathrm{C}}{16}+\left(\mathrm{B}-\frac{\mathrm{C}}{2}\right) t+\mathrm{C} t^{2}+8 \mathrm{c}
\end{array}
$$

Whereof the mean $=\mathrm{A}+\frac{\mathrm{C}}{16}+\mathrm{B} t+\mathrm{C} t^{2}$ \&c. For the mean of the twenty-four hours, we add $-t$ and $+t$, therefore

$$
\begin{gathered}
\text { General mean }=\frac{24 \mathrm{~A}}{24}+\frac{2 \mathrm{C}}{24} \Sigma_{1^{2}}^{12^{2}} \frac{t^{2}}{24^{2}}+\frac{2 \mathrm{E}}{24} \cdot \Sigma_{1^{4}}^{12^{4}} \cdot \frac{t^{4}}{24^{4}} \\
\quad=\mathrm{A}+\frac{650 \mathrm{C}}{12 \times 576}+\frac{60810 \mathrm{E}}{12 \times 576^{2}}=\mathrm{A}+\frac{\mathrm{C}}{11}+8 \mathrm{c} .
\end{gathered}
$$

Now C, D, \&c. being small, it is evident this nearly agrees with the homonymous mean, the chief error $\mathrm{B} t+\mathrm{C}\left(\frac{1}{16}-\frac{1}{11}=\frac{5}{176}\right)$ indicating ${ }^{\text {very }}$ nearly a progressively uniform error, so that by combining $t$ and $-t$ this error $=\frac{5}{176} \mathrm{C}=\frac{1}{35} \mathrm{C}$ must very nearly vanish. 
These extremely general theoretical results are amply confirmed by the above-mentioned observations.

London, December 8, 1841.

S. M. D.

APPENDIX.

'These Leith observations give the temperature at

\begin{tabular}{|c|c|c|c|c|c|c|}
\hline$h^{\mathrm{M}}=51 \cdot 149$ & & $=49.544$ & A.1 & $=46 \cdot 134$ & $\begin{array}{c}\text { A.M } \\
7\end{array}$ & $=4$ \\
\hline & 8 & & 2 & & 8 & \\
\hline & 9 & & 3 & & 9 & \\
\hline & 10 & & 4 & & 10 & \\
\hline & 11 & & 5 & & 11 & \\
\hline $50 \cdot 2$ & 1 & & & $45 \cdot 653$ & 12 & \\
\hline
\end{tabular}

The sums of the homonymous hours are-

\begin{tabular}{|c|c|c|c|c|c|}
\hline \multicolumn{2}{|c|}{$\begin{array}{l}\text { P.M. and A.M. } \\
1 \mathrm{~h}^{\mathrm{r}}=97 \cdot 283\end{array}$} & \multicolumn{2}{|c|}{$\begin{array}{l}\text { P.M. and A.M. } \\
\quad 7 \mathrm{~h}^{\mathrm{r}}=95.827\end{array}$} & \multirow{2}{*}{$\begin{array}{c}\text { Sums. } \\
193 \cdot 110 \\
193 \cdot 056\end{array}$} & \multirow{2}{*}{$\begin{array}{c}\text { Diff. } \\
+1 \cdot 456 \\
+1 \cdot 750\end{array}$} \\
\hline 2 & $97 \cdot 403$ & 8 & $95 \cdot 653$ & & \\
\hline & & 9 & & & 333 \\
\hline & & 10 & & & 400 \\
\hline & 96.266 & 11 & & & $-0 \mathrm{~T}$ \\
\hline & $95 \cdot 9$ & 12 & & 193.122 & $1 \cdot 2 c$ \\
\hline
\end{tabular}

The near agreement in the third column shows the series expressing the daily temperature to be very nearly a periodic one, and of the form $h=\mathrm{H}+\mathrm{A} \sin t+a \cos t+\mathrm{B} \sin 2 t$ $+b \cos 2 t+\mathrm{C} \sin 3 t+c \cos 3 t+\mathrm{E} \sin 4 t+e \cos 4 t ; h$, $\mathrm{H}$, \&c. being thermometric degrees, and $t$ the time.

Hence, as in my paper on the Plymouth barometric oscillations*, we can deduce the rule, that if the thermometer be observed only four times a day, at intervals of six hours, commencing at any time, the resulting average is all but equal to that deducible from twenty-four hourly observations. The greatest difference is here $48^{\circ} .266$ (mean) $-\frac{1}{4}\left(192^{\circ} .976\right)$ $=0^{\circ} .022=$ one forty-fifth of a degree of Fahrenheit.

The differences of the homonymous hours (P.M. - A.M.) are

$$
\begin{aligned}
& 1 \mathrm{~h}^{\mathrm{r}}=+5 \cdot 015\left|4 \mathrm{~h}^{\mathrm{r}}=+5 \cdot 795\right| 7 \mathrm{~h}^{\mathrm{r}}=+3 \cdot 161 \mid 10 \mathrm{~h}^{\mathrm{r}}=-1 \cdot 736 \\
& 2+5.5375+5.478|8 \quad+1.595| 11 \quad-3.147 \\
& 3+5.8436+4.641|9 \quad-0.226| 12 \quad-4.379
\end{aligned}
$$

Whence by a process exactly similar to the one in the paper above alluded to, there results

temp. from noon $=h=48^{\circ} \cdot 266+2^{0} \cdot 1497 \sin t+2^{\circ} \cdot 1354 \cos t$ $+0.295 \sin 2 t+0.308 \cos 2 t-0.1302$

* Phil. Mag., June 1842 (Third Series, vol. xx. p. 477). 


$$
\begin{aligned}
& \begin{array}{l}
\sin 3 t+0^{\circ} 00115 \cos 3 t-0.00715 \sin 4 t \\
+0.00686 \cos 4 t
\end{array} \\
&\left.\begin{array}{rl}
\text { temp. from } \\
\text { noon }=h
\end{array}\right\}=48^{\circ} .266+3^{\circ} .0257 \sin \left(t+44^{\circ} 53^{\prime}\right)+0^{\circ} .4265 \\
& \sin \left(2 t+46^{\circ} 14^{\prime}\right)+0^{\circ} \cdot 1302 \sin (3 t \\
&\left.+179^{\circ} 30^{\prime}\right)+0.0099 \sin \left(4 t+136^{\circ} 50^{\prime}\right)
\end{aligned}
$$

The quantities $c, \mathrm{E}$, and $e$ are the only ones wherein the separate values in each combination disagree, but this is not very material, owing to the smallness of these quantities.

London, April 29, 1842.

S. M. D.

X1. On the Motion of Luminous Waves in an Elastic Meditum, consisting of a system of detached particles, separated by finite intervals. By S. Earnshaw, M.A. of St. John's College, Cambridge.

TTHE equations obtained at the close of my last communication on this subject (vol. xx. p. 373) involve six coefficients, A, B, C, D, E, F. From the peculiar manner in which they enter those equations it is known, that if the coordinate axes be turned through proper angles, their directions still remaining rectangular, the equations will assume the forms

$$
d_{t}^{2} \xi=-k_{1}^{2} \xi, \quad d_{t}^{2} \eta=-k_{2}^{2} \eta_{3} \quad d_{t}^{2} \zeta=-k_{3}^{2} \zeta .
$$

These show that vibrations of $m$ parallel to any one of the axes of dynamical symmetry cannot be affected by vibrations which are parallel to the other axes. Simple as these equations are, they have precisely the same degree of generality as the original ones, for the motion of the particle $m$. It might not happen that the axes of dynamical symmetry for every particle would be parallel to those for $m$, and that the same position of the coordinate axes would reduce the equations of motion for the other particles of the medium to the same form, and cause them to have the same coefficients as for $m$. A condition equivalent to mechanical homogeneity of the medium must be fulfilled that this may be the case. It is necessary therefore to appeal to experiment for license in this matter. By experimental means we learn that the positions of the axes of elasticity for waves of a given length are fixed, and that the velocity of transmission of such waves is uniform, and that both these properties are independent of the thickness of the medium : hence we may assume that $k_{1} k_{2} k_{3}$ have constant values through the whole interior of a medium, and that the equations in the simple forms above given are applilicable to, and fully represent all the properties of, the trans- 